\title{
Novel Techniques for Fair Rate Control in Wireless Mesh Networks
}

\author{
Mohiuddin Ahmed \\ Computer Science and Engineering Department \\ Islamic University of Technology \\ Gazipur, Bangladesh
}

\begin{abstract}
IEEE 802.11 based wireless mesh networks can exhibit severe fairness problem by distributing throughput among different flows originated from different nodes. Congestion control, Throughput, Fairness are the important factors to be considered in any wireless network. Flows originating from nodes that directly communicate with the gateway get higher throughput. On the other hand, flows originating from two or more hops away get very little throughput. For this reason a distributed fair scheduling is an ideal candidate for fair utilization of gateway's resources (i.e., bandwidth, airtime) and thereby achieving fairness among contending flows in WMNs. There are numerous solution for aforementioned factors in wireless mesh network. We figured out some problems of few existing solutions and integrated to give the solution for those problems. We considered neighborhood phenomenon, airtime allocation and elastic rate control to design a novel technique to achieve fair rate control in wireless mesh network. And finally we introduce distributed fair scheduling to get fairness in mesh network.
\end{abstract}

Keywords-Wireless mesh network; Network Throughput; Congestion Control; Fairness; Airtime Allocation; Neighbourhood Phenomenon; Gateway. (key words)

\section{INTRODUCTION}

Wireless Mesh Networks [1] (WMNs) are envisioned to replace the wired backbone by multi hop wireless network. The shared nature of the wireless medium, unpredictable link quality, and existence of hidden terminals pose a number challenges for WMNs. The poor performance of TCP in wireless networks demands efficient congestion control in WMNs. Furthermore, efficient rate control is required for fair bandwidth sharing and optimum network throughput. We work toward designing a distributed fair rate control mechanism in WMNs.

As various wireless networks evolve into the next generation to provide better services, a key technology, wireless mesh networks (WMNs), has emerged recently. In WMNs, nodes are comprised of mesh routers and mesh clients. Each node operates not only as a host but also as a router, forwarding packets on behalf of other nodes that may not be within direct wireless transmission range of their destinations. A WMN is dynamically self- organized and self-configured, with the nodes in the network automatically establishing and maintaining mesh connectivity among. This feature brings many advantages to WMNs such as low up-front cost, easy

\author{
K.M.Arifur Rahman \\ Electrical \& Electronic Engineering Department \\ Bangladesh University of Engineering and Technology \\ Dhaka, Bangladesh
}

network maintenance, robustness, and reliable service coverage.

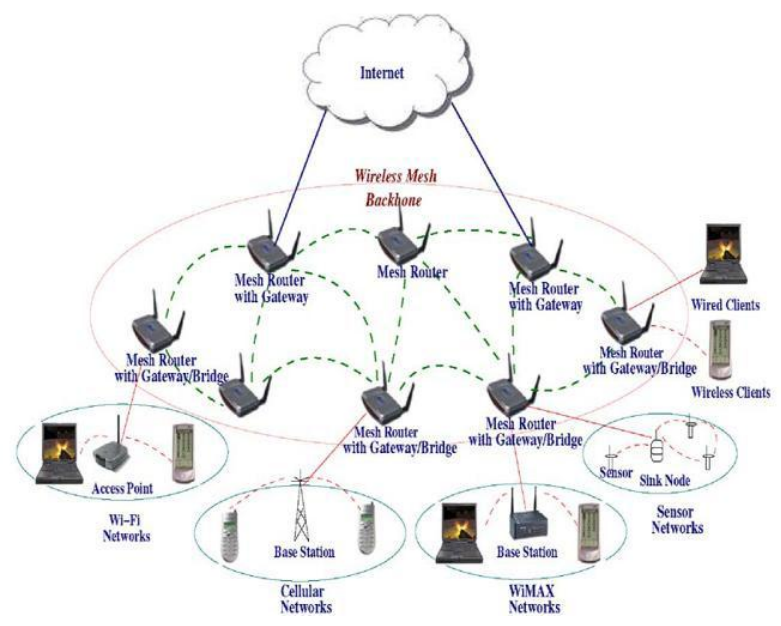

Figure 1. Wireless Mesh Network

WMNs consist of two types of nodes: mesh routers and mesh clients.Fig. 1 shows a basic wireless mesh network provided by Other than the routing capability for gateway/repeater functions as in a conventional wireless router, a wireless mesh router contains additional routing functions to support mesh networking. To further improve the flexibility of mesh networking, a mesh router is usually equipped with multiple wireless interfaces. Compared with a conventional wireless router, a wireless mesh router can achieve the same coverage with much lower transmission power through multi hop communications. Optionally, the medium access control (MAC) protocol in a mesh router is enhanced with better scalability in a multi-hop mesh environment.

A major challenge of IEEE 802.11 based wireless mesh network is to provide fair rate allocation. In multi-hop WMN nodes that are directly connected to the gateway get higher priority for their aggregate flow than the nodes that are two or more hops away from the gateway. This results in severe unfairness and causes starvation of flows travelling multiple hops. So, a fair rate control mechanism is needed to control the high priority traffic and to allow multi hop nodes to increase their rate.

Unfairness is considered as the consequence of one of the pressing issues of hidden node problem. Hidden terminal 
interference is caused by the simultaneous transmission of two node stations that cannot hear each other, but are both received by the same destination station. It is said that RTS/CTS can solve the problem but still there exists the problems of low throughput and increased average packet latency and poor fairness.

In this paper, we analyzed the solutions from three research papers $[2,3,4]$ and then provided our approaches to eliminate those problems. Our contribution includes the elimination of stealing effect, maximum airtime allocation and mini backup. And thus our approaches are capable of ensuring fair rate control mechanism in wireless mesh network.

\section{RELATED WORK}

Wireless mesh network has become an attractive research area now-a-days. Significant research has been done to investigate the rate and congestion control of wireless multi hop networks.

In GAP [2] there is a rate control mechanism which limits rate of single hop nodes by enforcing a utilization threshold.
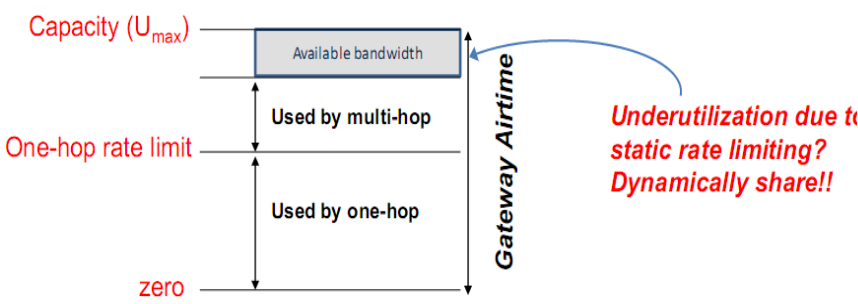

Figure 2. Gateway Airtime Partitioning

They considered traffic only to and from gateway. Thus their proposed algorithm only tries to provide fair share for all spatially disadvantaged nodes around the gateway, not in anywhere else. So this algorithm is not well suited in such network where local traffic exists.

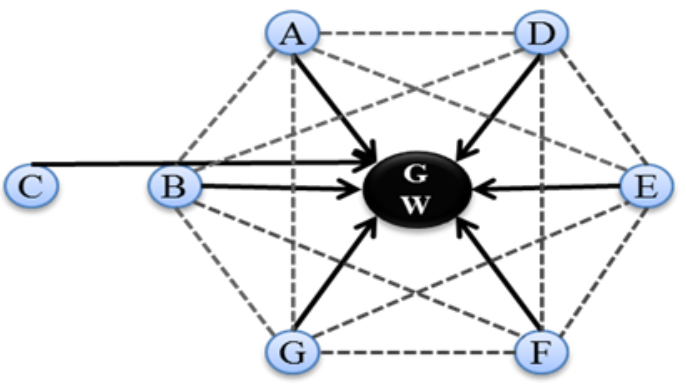

Figure 3. Multiple Competing Flows

Their considered network holds single hop node as leaf node. That creates a hidden terminal problem.

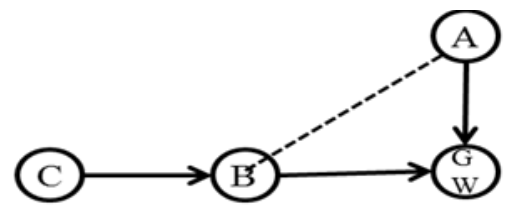

Figure 4. Single competing flow
Considering Figure. 4, whenever A gains the network then $\mathrm{B}$ will not get chance and thus nodes connected with $\mathrm{B}$ will be congested and A will get high priority always among its one hop competing flows. As usual data transmission will require more time and thus the congested nodes data transmission will lead to a worse situation.

In addition, IFA [5] and [6] proposed link-layer rate control mechanism to solve TCP fairness. In IFA the authors studied per-TAP fairness and end-to-end performance in WMNs (Multi-hop wireless backhaul networks).They propose an interTAP fairness algorithm that aims to achieve per-TAP fairness without modifying the TCP protocol. Here, nodes explicitly exchange information and all nodes calculate their fair shares using that information. Also algorithm proposed by Raniwala et al. [6] works away from the unstable MAC layer.

Alternatively, another class of work to rate and congestion control is done by Rangwala et al. where wireless congestion is considered as neighborhood phenomenon [4] instead of considering as per flow problem.

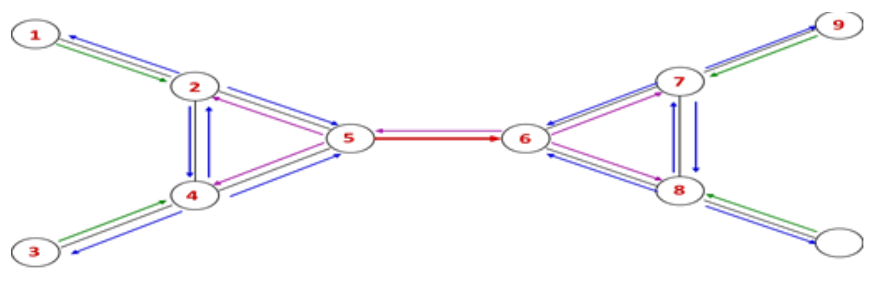

Figure 5. Neighborhood Phenomenon

According to these schemes when a link is congested then all the correspondent neighbor link has to reduce their transmission rate to solve the congestion of that link. As a consequence the neighbor link's neighbor also has to limit their rate although those links are not supposed to reduce their rate because their transmission doesn't affect the root congested link. And thus the network utilization and throughput is reduced. They consider mesh network as independent network and Converging \& diverging of flows are absent here.

Furthermore, Ramesh Govindan et al. focused on airtime allocation [3] to solve the drawbacks of [4]. In [3] they assigned minimum airtime limit to each of the active link in the network. The calculation of the assigned airtime limit is based on the number of active neighborhood link. These assigned airtime limit converges very slowly from minimum to optimal. Moreover, the calculation of the airtime limit is sophisticated due to its control overhead.

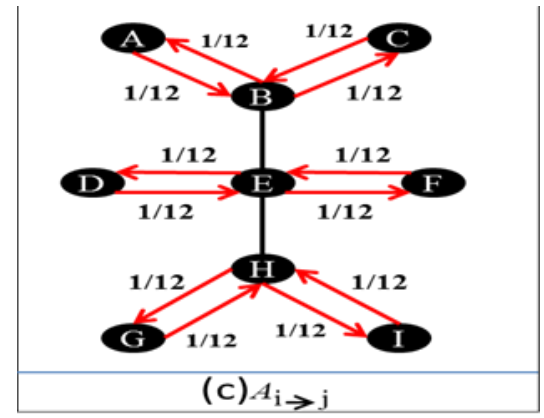

Figure 6. Airtime Allocation 
In MLM-FQ [8] local schedulers self-coordinate their scheduling decisions and collectively achieve fair bandwidth sharing. But it does not allow spatial channel reuse of network resources. Same authors then proposed EMLM-FQ to further improve the spatial channel reuse. Moreover both the scheduling techniques are sender initiated thus have good probability of collisions.

\section{PROBLEM DESCRIPTION}

\section{A. Gateway Airtime Partitioning[2]}

Define abbreviations and acronyms the first time they are used in the text, even in this paper [2] they considered traffic only to and from gateway \& thus their proposed algorithm only tries to provide fair share for all spatially disadvantaged nodes around the gateway, not in anywhere else. So this algorithm is not well suited in such network where local traffic exists.

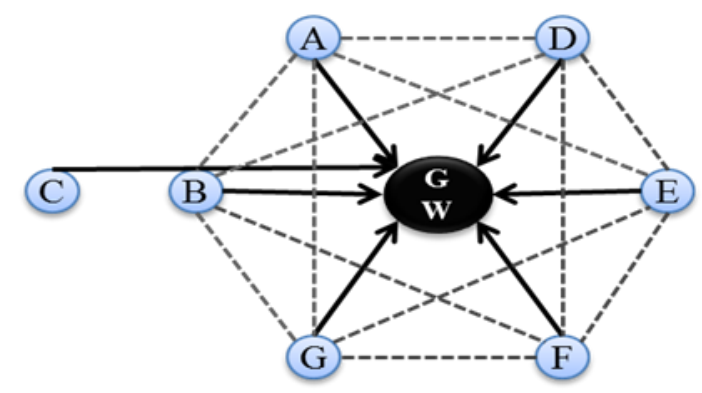

Figure 7. Two-hop node competing with single-hop node \& Dotted lines indicate that connected nodes are within sensing range

Their considered network holds single hop node as leaf node. That creates a hidden terminal problem (Stealing Effect). Previous papers bypassed the stealing effect scenario \& no solution was provided.

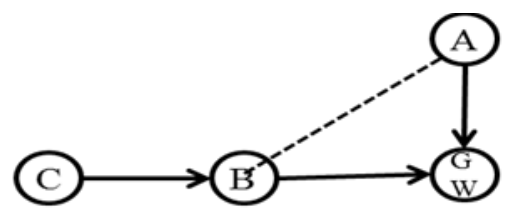

Figure 8. Oversimplified Network Model

The single hope leaf node connected to gateway doesn't sense the other node as it is out of the interference range of each other so one node will be able to transmit and other will get chance when one finishes.

Considering figure.8, whenever A gains the network then B will not get chance and thus nodes connected with $\mathrm{B}$ will be congested and A will get high priority always among its one hop competing flows. As usually data transmission will require more time and thus the congested nodes data transmission will lead to a worse situation. Their considered network model is oversimplified as single hop nodes are leaf node. It is very usual that there might be some other scenarios of network where single hop node is not the leaf node. If we consider the different scenarios like mentioned then the oversimplified problem can be solved.
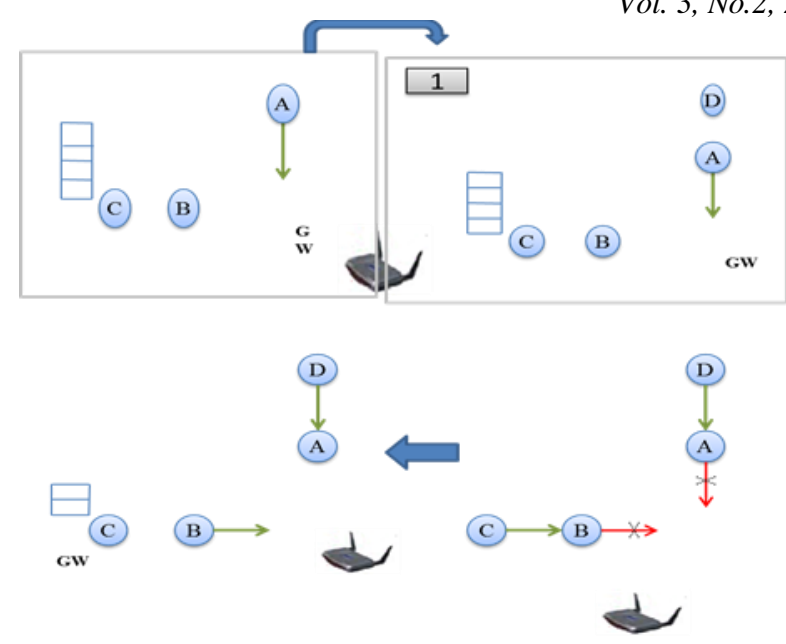

Figure 9. Modified Topology

Now we see that in the above figure 9. Whenever D will gain the network A have to stop sending data to gateway and thus creates an opportunity for other competing flows who were congested earlier. Once the disadvantaged nodes get chance others will not be able gain the network resource unless they finish. Thus short term fairness might not be possible but long term fairness is easily achieved. But the scenario is not beyond Stealing Effect problem. Stealing effect might occur in any depth of the network among two hop away nodes.

\section{B. Transparent Airtime Allocation [3]}
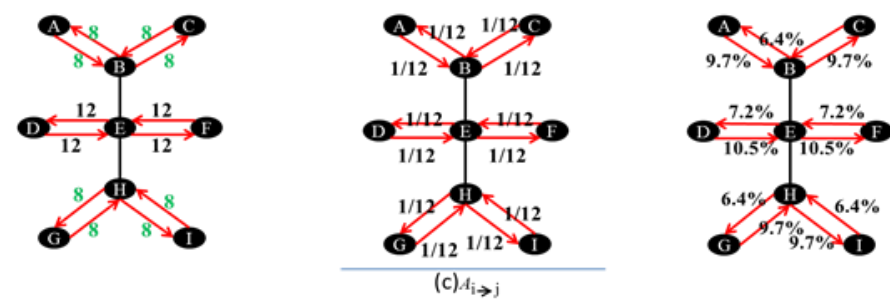

Figure 10. Airtime Allocation

In this paper [3] the writer focused on airtime allocation to solve the drawbacks of the previous paper e.g. Network Underutilization. They assign minimum airtime limit to each of the active link in the network. The calculation of the assigned airtime limit is based on the number of active neighborhood link. These assigned airtime limit converges very slowly from minimum to optimal. Moreover the calculation of the airtime limit is sophisticated due to its control overhead.

\section{Understanding Congestion Control[4] Understanding Congestion Control[4]}

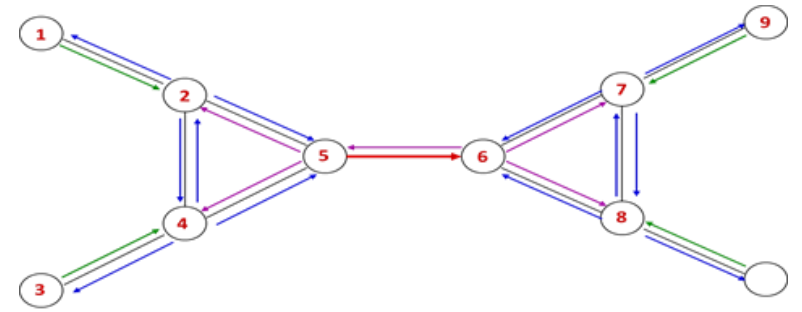

Figure 11. Considered Network Topology. 
They considered congestion control as neighborhood phenomenon. When a link is congested then all the correspondent neighbor link has to reduce their transmission rate to solve the congestion of that link.

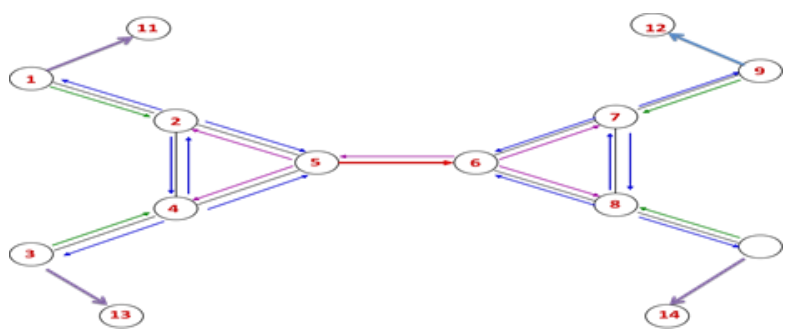

Figure 12. Different Network Topology

As a consequence the neighbor link's neighbor also has to limit their rate although those links are not supposed to reduce their rate because their transmission doesn't affect the root congested link. And thus the network utilization and throughput is reduced. They consider mesh network as independent network and Converging \& diverging of flows are absent here. Gist is they fail to provide proper network utilization.

\section{Proposed TeChniques}

Our solution is comprised of three main components. We first find the maximum air time limit by constructing the compatibility graph, which contains the links that can be activated at the same time and define different possible cliques (see Subsection A),then we use a distributed fair scheduling mechanism by Start Time Fair Queuing (see Subsection B). Finally, we solve the stealing effect by receiver initiated CTS mechanism (see Subsection C).

\section{A. Assignment of Maximum Airtime Limit}

In paper [3], airtime was assigned to each link dynamically from minimum to maximum (discussed earlier). Our challenge is to assign maximum airtime limit to each active link of the network. We define a procedure to compute the airtime-limit, by constructing compatibility graph depending on the neighborhood scenario of the network and find different possible cliques.

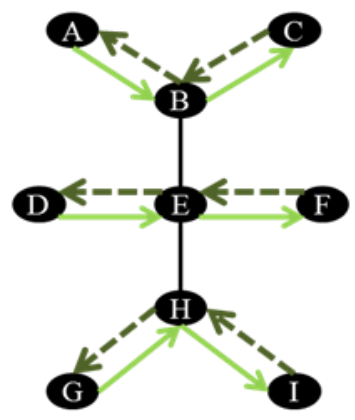

Figure 13. Topology of the network

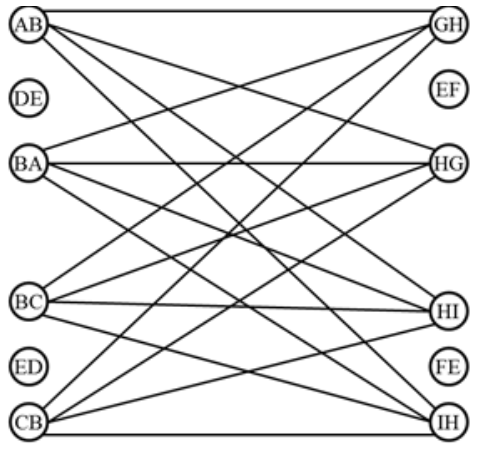

Vol. 3, No.2, 2012

Figure 14. Compatibility graph of the network presented in figure 13

Here in the compatibility graph, each vertex represents single active link $\{i, j\}$ of the network and the edge between two vertices tell that these two links can be activated at the same time.

Now from the above compatibility graph we can construct the set of all possible cliques for the corresponding network, where a clique is a set of all links that can be activated simultaneously. A clique is a complete sub graph. From the figure 14 we get 8 sets of cliques like below-

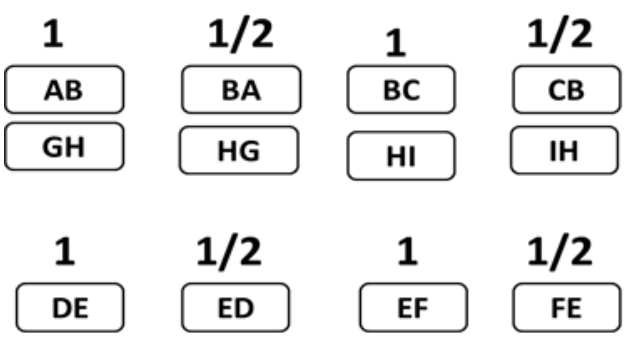

Figure 15. Clique sets for figure 14

- Weight of Data traffic $=1$

- Weight of ACK traffic $=1 / 2$

- $1+1 / 2+1+1 / 2+1+1 / 2+1+1 / 2=6$

- So, 6 different time slots are required

So, from the above scenario it is clear that, maximum $1 / 6$ of the total airtime limit will be assigned to each clique set and we can show it like below-

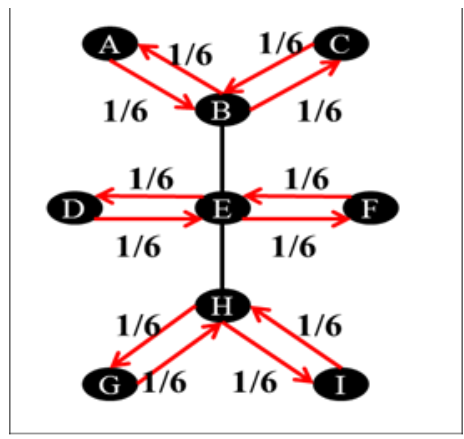

Figure 16. Maximum Airtime Limit to each flow

Thus our second challenge is solved by assigning maximum $1 / 4$ of the airtime limit to each flow of the network. 


\section{B. Start Time Fair Queuing}

We propose a distributed fair scheduling mechanism- Start Time Fair Queueing to achieve fairness and to minimize collision in the mesh network. Here each node will maintain a table where there are two fields - start tag and finish tag. Every node shares their start tag. When a node transmits it include the finish tag of next packet in its header.

For example- node 1 has following start tags and finish tags for their packets-

\begin{tabular}{|l|l|}
\hline \multicolumn{1}{|c|}{ Start Tag } & \multicolumn{1}{c|}{ Finish Tag } \\
\hline 100 & 130 \\
\hline 130 & 150 \\
\hline 150 & 190 \\
\hline
\end{tabular}

TABLE 1. StART TAg AND Finish TAg For A PACKET

Here, first packet has start tag 100, packet size 30. So its finish tag is 130. Second packet has start tag 130, packet size 20. So its finish tag is 150 . Third packet has start tag 150 , packet size 40. So its finish tag is 190 . When node 1 transmits packet 1 to other node (i.e., node 2) it include the finish tag of packet 2 (i.e., here 150) in its header. All other nodes of node 1 's transmission range will overhear this information and know its next packet's finish time. In this way each node will know the finish time of next packet of all other nodes belong to its transmission range. This information will be in the sorted order in the table.

Node with the lowest finish time above in the table will transmit next. Whenever any node finishes its transmission its finish time will be higher immediately and it will not transmit any packet for long time. Next node with the lowest finish time above in the table will transmit and update its finish tag value. This process goes on until all the packets are transmitted.

One problem of this mechanism is that -if finish tags of two node become equal, then collision occurs. To solve this problem we propose another mechanism - Mini Backup, based on maximum airtime limit.

In mini backup we prioritize a flow based on the maximum airtime limit that we proposed in previous section 4.1. Here the flow that has already done more airtime utilization will get less priority and flow that has done less airtime utilization will get higher priority. But still each node may experience collision due to stealing effect caused by hidden node and may starve for long time. For this we need to solve the stealing effect.

\section{Elimination of the Stealing Effect}

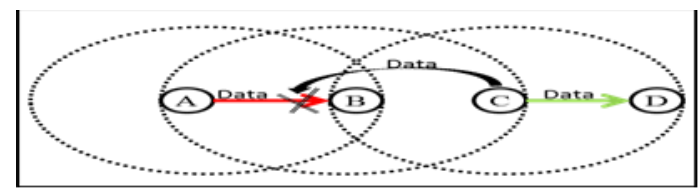

Figure 17. Hidden Node Problem

Here, node A will try several times to send its data and each time the data packets sent by $\mathrm{C}$ could collide with the data packets sent by A. Collision occur due to contention with hidden nodes(detail of this problem is discussed in section) and node A will starve.
We introduce receiver initiated CTS mechanism here to solve the above problem. In this mechanism, receiver (i.e., here node B) takes the responsibility to send CTS periodically to all the nodes of its transmission range (i.e., here node $\mathrm{A}$ and $\mathrm{C}$ ) when it senses a collision within its transmission range.

So, after getting CTS node C will stop transmitting data to node $\mathrm{D}$ and node $\mathrm{A}$ will get the chance to transmit data. Thus starvation of node A will be removed.

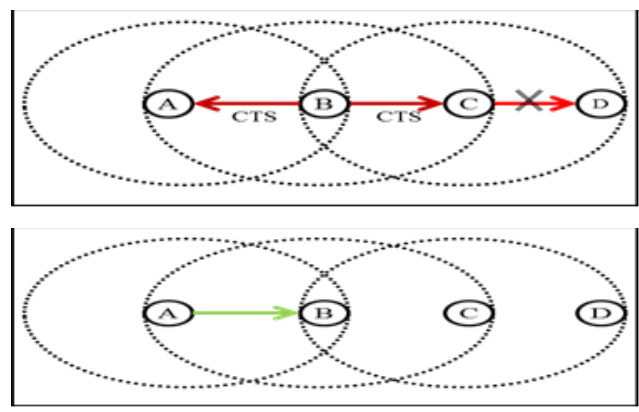

Figure 18. Solved scenario of Hidden Node Problem

\section{CONCLUSION}

In our research paper, we integrated the ideas from different research works and proposed some new approaches for fair rate control. Basically, we proposed new techniques for maximum airtime allocation, elimination of stealing effect and start time fair queuing. All these approaches are efficient enough to ensure fairness in wireless mesh network. In future, we will work extensively on these issues and would like to find out more efficient solutions along with real time simulation results.

\section{REFERENCES}

[1] Ian F. Akyildiz, Xudong Wang, Weilin Wang, Wireless mesh networks: a survey, Computer Networks (2005)

[2] Vincenzo Mancuso, Omer Gurewitz, Ahmed Khattab and Edward W. Knightly, Elastic Rate Limiting for Spatially Biased Wireless Mesh Networks, ACM MobiCom (2010).

[3] Ki-Young Jang, Konstantinos Psounis, Ramesh Govindan, Simple Yet Efficient, Transparent Airtime Allocation for TCP in Wireless Mesh Networks,CoNEXT (2010)

[4] RANGWALA, S., JINDAL, A., JANG, K.-Y., PSOUNIS, K.,AND GOVINDAN,R.Understanding congestion control inmulti-hop wireless mesh networks. In Proc. of ACM MobiCom (2008).

[5] V. Gambiroza, B. Sadeghi, and E. W. Knightly, "End-to-End Performance and Fairness in Multi-hop Wireless Backhaul Networks," in Proceedings of ACM MOBICOM, Philadelphia, PA, USA, Sept. 2004.

[6] A. Raniwala, P. De, S. Sharma, R. Krishnan, and T. c. Chiueh, "End-toend flow fairness over ieee 802.11-based wireless mesh networks," in Proceedings of IEEE INFOCOM,2007

[7] Haiyun Luo, Jerry Cheng, and Songwu Lu, "Self-Coordinating Localized Fair Queueing in Wireless Ad Hoc Networks",IEEE ToM,04

\section{AUTHORS PROFILE}

Mohiuddin Ahmed has achieved Bachelor of Science degree in Computer Science and Information Technology from Islamic University of Technology. Research interest includes Human Computer Interaction, Cloud Computing, Wireless Networks, Artifical Intelligence.

K.M.Arifur Rahman is a final year undergraduate student in Electrical and Electronic Engineering department in Bangladesh University of Engineering and Technology. Research interest includes Genetic Algorithm, Ubiquitous Computing, Wireless Network and Image Processing. 